\title{
Panorama del servicio de referencia digital en bibliotecas de la UNAM
}

\author{
Bardo Javier García Martínez
}

\begin{abstract}
Resumen
Actualmente se observa una marcada disparidad de la presencia en línea de varias bibliotecas universitarias pertenecientes a la Universidad Nacional Autónoma de México (UNAM). Este hecho representa una problemática notoria cuando se toma en cuenta el indicador sobre el acceso a estos servicios digitales. El presente trabajo considera 35 bibliotecas de dicha universidad, vinculadas al Subsistema de Licenciatura y Posgrado, con el objetivo de observar la estructura de sus sitios web. Esto con el fin de enfocarse en los posibles medios de acceso que tienen los usuarios de esta comunidad bibliotecaria a un servicio de referencia digital. Para ello, se parte primero de la conceptualización del servicio de referencia, así como sus tendencias actuales, para poder conocer los parámetros mínimos que se espera de un servicio de esta naturaleza. Posteriormente, se indaga sobre las particularidades (tanto ventajosas como desventajosas) en términos de estructura y organización, que componen dichos accesos respecto a una posible referencia digital y el estado actual en que se encuentran. Finalmente, se culmina con una reflexión alineada con la metodología aquí explorada.
\end{abstract}

Palabras clave: bibliotecas universitarias, bibliotecas digitales, sitios web, servicio de referencia, referencia digital.

\section{OVERVIEW OF THE DIGITAL REFERENCE SERVICE AT UNAM LIBRARIES}

\begin{abstract}
There is currently a wide disparity of features in the online versions of many college libraries under the administration of the National Autonomous University of Mexico (UNAM). This problem is particularly pervasive in aspects dealing with access to digital services. That is why in this article 35 libraries of the undergraduate and graduate programs subsystem were analyzed in order to understand their underlying website structures. This was done by focusing on the possible means of access that users of the library community have to a digital reference service. In order to achieve this, we began by conceptualizing the referral service, as well as its current trends, in order to meet the minimum standards expected of a service of this nature. Subsequently, we researched their particularities, in terms of structure and organization of such accesses, regardless of their advantages or disadvantages. Finally, we carry out a reflection based on this methodology.
\end{abstract}

Keywords: faculty libraries, digital libraries, websites, reference service, digital reference

Recepción: 02/04/2020. Aprobación: 05/08/2020. Dol: http://doi.org/10.22201/cuaieed.16076079e.2020.21.6.12 
Doctor en Bibliotecología y Estudios de la Información en la Universidad Nacional Autónoma de México (UNAM), así como Maestro (EDINBA) y Licenciado (UNITEC) en Diseño Gráfic; cuenta con la Especialidad Advanced Web Design. Labora en el departamento de Publicaciones de la Dirección General de Bibliotecas (DGB), UNAM. Su experiencia y conocimientos versan principalmente sobre al ámbito editorial enfocado a las revistas académicas electrónicas y los libros electrónicos; adicional a ello tiene una amplia experiencia desarrollada en el campo del diseño impreso, web y multimedia. Actualmente labora en el departamento de Publicaciones, Secretaría Técnica de Difusión y Relaciones de la DGB, UNAM.

\section{Introducción}

El servicio de referencia cuenta con una serie de propósitos y alcances determinados que, como cualquier otro servicio del ámbito bibliotecario, ha evolucionado en función del avance de las tecnologías que envuelven a la comunidad que atiende. Su presencia actual dentro del espacio universitario se manifiesta en la forma de una referencia digital, y el presente trabajo tiene como objetivo realizar un esfuerzo por explorar la situación del acceso a dicho servicio durante el año 2019, dentro de la presencia web del Subsistema de Licenciatura y Posgrado dentro de la Universidad Nacional Autónoma de México (UNAM).

Además, se planteó encontrar las características más relevantes y el funcionamiento de las herramientas que permitan exponer las buenas y malas prácticas dentro de este caso de estudios. Este trabajo nace debido a la gran relevancia que tiene este servicio en las necesidades especializadas según cada carrera universitaria, pues resulta medular en la búsqueda de información y solución de problemas propios la vida estudiantil universitaria.

\section{Servicio de referencia}

La referencia en bibliotecas está relacionada directamente con el acceso a la información. Su objetivo es guiar al público hacia las formas más eficientes de obtener la información que requieren, no sólo haciéndola asequible, sino también instruyendo al usuario en los métodos correctos para llegar a ella. La American Library Association ofrece una definición del servicio de referencia:

Bibliotecarios que asisten, asesoran e instruyen a los usuarios para el acceso a todas las formas de conocimiento registrado. La asistencia, el asesoramiento y la instrucción incluyen tanto el servicio directo como el indirecto a los usuarios (Reference and User Services Association [RUSA], 2017). 
"Panorama del servicio de referencia digital en bibliotecas de la UNAM"

Bardo Javier García Martínez

Vol. 21, Núm. 6, noviembre-diciembre 2020

Revista Digital Universitaria

Es un deber del referencista enseñar el uso apropiado de los recursos de información, solventar con precisión las dudas de los usuarios, proveer información sobre las fuentes que puedan servir para que éstos solucionen sus propias dudas, seleccionary recomendar materiales acordes a las necesidades de información de los distintos tipos de usuarios, así como impulsar las habilidades informativas de la comunidad a la que atiende. De acuerdo con González:

El servicio de referencia se presta por distintos canales, tales como teléfono, chat, redes sociales (referencia inmediata), correo electrónico, formulario Consúltenos y FAQS (referencia no inmediata) y, sobre todo, la atención en persona al usuario en los mostradores y puntos de información situados en las salas de lectura de las bibliotecas y en las áreas de trabajo del personal bibliotecario (González, 2012, p. 569).

\section{Tendencias actuales en el servicio de referencia}

En el espacio universitario, la referencia es un servicio vital debido a que el nivel de estudios que requiere un usuario en este ámbito corresponde a necesidades de información amplia y, en muchos casos, estas necesidades son especializadas.

Aunque el perfil de un usuario de biblioteca universitaria incluye a estudiantes y profesores, al comparar el número de estudiantes con el de profesores se hace evidente que el grueso de los usuarios se encuentra del lado estudiantil. Si se contempla este perfil, se puede señalar que una de sus características más importantes radica en la facilidad para el uso de la tecnología más actual; ya sea en computadoras portátiles, tabletas o teléfonos inteligentes. De esta manera, se entiende que los lugares más probables para que los estudiantes universitarios busquen un servicio de referencia pertenezcan al espacio digital.

La referencia digital es una consecuencia del empeño en el sector bibliotecario para adaptar las tecnologías disponibles y mejorar con ello sus servicios, para acercarse de manera proactiva a los usuarios. Según Martínez del Prado:

La referencia electrónica o digital ha sido el resultado de la evolución en las nuevas formas de acceder a la información, pues los años pasados han traído avances en las redes computacionales, trayendo consigo consecuencias inmediatas y permanentes para la provisión de servicios de referencia (Martínez del Prado, 2008, p. 20).

Las aplicaciones de este servicio en el ámbito digital han sido diversas, pero un medio electrónico actual que cobra mucha fuerza para atender estas necesidades se encuentra en las redes sociales. Ahí, el esquema de atención cambia porque ahora es la biblioteca quien se acerca a los usuarios y no son ellos los que tienen que "salir" a buscar dicho servicio. Aún considerando todos los cambios que han presentado, el servicio de referencia digital no pierde de ninguna manera las metas que debe cumplir, ya que debe buscar siempre solventar las dudas y consultas de los usuarios tomando como ventaja las tecnologías disponibles. 
"Panorama del servicio de referencia digital en bibliotecas de la UNAM"

Bardo Javier García Martínez

Vol. 21, Núm. 6, noviembre-diciembre 2020

Revista Digital Universitaria

Al respecto, la International Federation of Library Associations and Institutions (IFLA, 2002, p. 2) establece políticas claras y, por ello, sugiere tomar en cuenta los procedimientos existentes de la institución, la asignación de responsabilidades, así como definir y delimitar la clientela primaria a quien va dirigido este servicio.

Al tratarse de una sesión de preguntas y respuestas de manera remota y atemporal, se observa que se modifica la dinámica y se guía al usuario de manera distinta. Una gran ventaja de este cambio es que el usuario se toma el tiempo necesario para redactar su pregunta, dándole la oportunidad de estructurarla de mejor manera que si fuera una situación presencial. De esta forma, "El servicio de referencia digital se puede considerar como una entrevista de referencia en línea que puede durar menos de un minuto hasta el tiempo necesario para alcanzar una respuesta satisfactoria" (Katz, 2002, p. 2).

\section{Referencia digital en la UNAM}

La unAm es una institución educativa fundamental en México, pues se considera la cuna de la formación universitaria y de la investigación en el país. En este sentido, la actividad de referencia como apoyo en las labores académicas en los niveles de licenciatura y posgrado debe residir como un servicio prominente y bien estructurado. A partir de esta premisa se presenta aquí una indagatoria sobre la presencia de este servicio en el ámbito web, específicamente sobre los medios de acceso y contacto que tienen disponibles los usuarios en una búsqueda en su modalidad digital.

El subsistema de bibliotecas pertenecientes a licenciatura y posgrado dentro de la UNAM está constituido por veintiséis entidades, de las cuales se pueden encontrar un total de 35 bibliotecas. En algunos casos, no obstante, el sistema de posgrado llega a contar con el apoyo de bibliotecas de entidades colaboradoras, con sus respectivas Facultades, como pueden ser institutos de investigación o bibliotecas externas. A pesar de este arreglo, en el presente trabajo únicamente se tomarán en cuenta las 35 bibliotecas que son destinadas específicamente a cada entidad. Estas entidades son las responsables directas de los servicios a su comunidad. En la tabla 1 (ver anexo) se muestran a detalle las bibliotecas que componen este subsistema, ordenadas por entidades.

Dentro de la presencia web de las 35 bibliotecas mencionadas, se buscaron los medios y accesos más factibles en los que los usuarios pueden acudir a un servicio de referencia digital. Los canales digitales encontrados que califican para este fin fueron: a) cuentas de correo electrónico de contacto, b) formularios de contacto, c) redes sociales, y d) un caso especial de chat en la Biblioteca Digital de la Universidad (BiDi-unam).

Las modalidades que se mencionaron como potencial contacto para referencia digital se distribuyen como se muestra en la imagen 1. Hay que señalar que en algunas bibliotecas se repite más de una opción. Por otra parte, dentro de 
Imagen 1. Acceso potencial a servicio de referencia digital en bibliotecas de licenciatura y posgrado, unam. Fuente: elaboración propia.

Imagen 2. Ejemplos de chat social preparado en bibliotecas de la unam. Fuente: cuentas de Facebook de las bibliotecas de la Facultad de Ciencias y "Lino Picaseño", 2019.

la tabla 2 (ver anexo), se desglosan las características específicas que pueden servir para implementar el servicio de referencia en cada modalidad. En la tabla 3 (ver anexo), se señala su división respecto a si cuentan con un contacto de manera textual o mediante un sistema predefinido de correo. En lo que concierne a las bibliotecas que cuentan con redes sociales para establecer contacto, en la tabla 4 (ver anexo), se observa una clara división según el tipo de acceso a dichas redes.

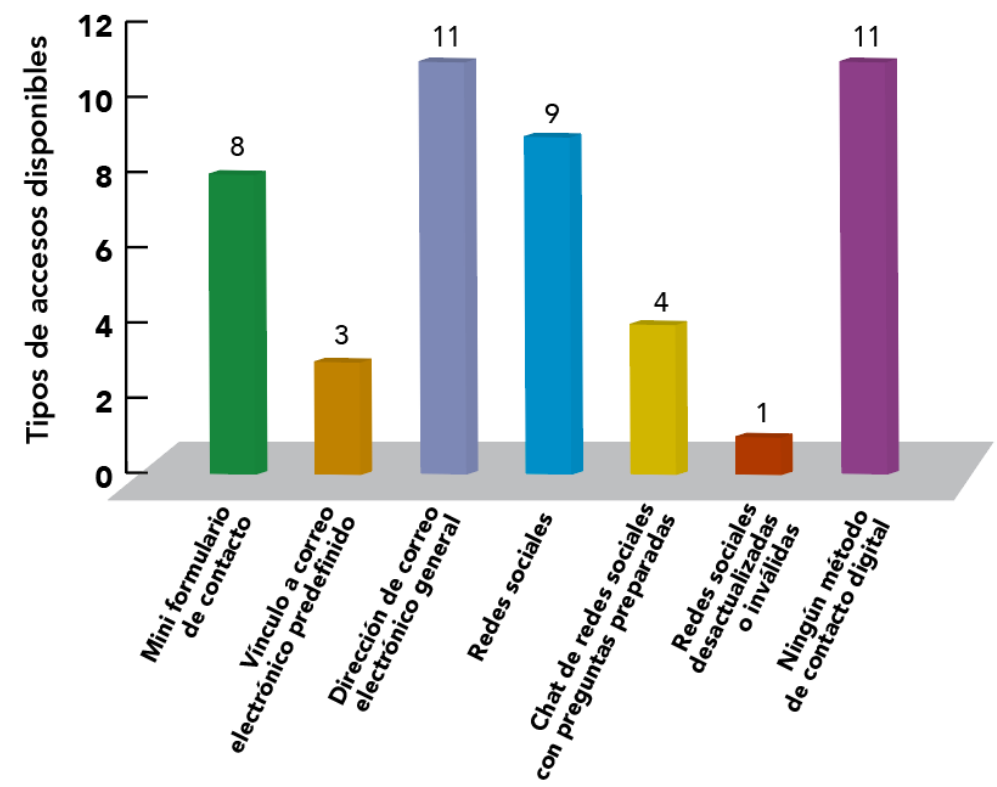

De acuerdo con la situación actual en la que se encuentran dichas bibliotecas, el mayor potencial que tienen para un servicio de referencia digital reside en el contacto mediante redes sociales con preguntas automatizadas. Un ejemplo de ello se observa en la imagen 2, en donde la biblioteca "Lino Picaseño" y la Facultad de Ciencias manejan esta comunicación por un chat social. Hay que señalar que este tipo de presencia ante el usuario puede

\section{递 Facultad de Ciencias}

A 52 mil personas les gusta esto incluidos Elke Vargas y 7 amigos Universidad

Normalmente responde en algunas horas

21. A 52 mil personas les gusta esto, incluidos Elke Vargas y 7 amigos
Biblioteca Lino Picaseñ...

\section{$\Xi$ Normalmente responde en un día \\ (i) Elige una opción o escribe un mensaje.}

¿Cuánto cuesta esta escuela?

Quiero más información sobre esta escuela.

Aceptan nuevos estudiantes?

¿Dónde están?
X oportunidad para hacerle sugerencias y abordar las dudas más comunes, al mismo tiempo que se deja la opción abierta a consultas más amplias.

En algunos casos, se apoya la formalidad del servicio cuando se especifican previamente los horarios de atención. Desafortunadamente, esta opción de servicio es en su mayoría desaprovechada debido a los tiempos de respuesta. Asimismo, las preguntas automáticas versan sobre temáticas generales y, de entrada, ya están Escribe un mensaje...

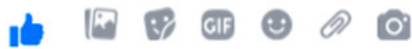

1 disponibles en la sección de información de su página institucional en Facebook. 


\section{Discusión sobre las problemáticas de la referencia actual}

El problema con la referencia digital en este subsistema de la unAm es que está casi ausente, pues, en la mayoría de los casos, no es parte formal de la sección de servicios. A pesar de ello, algunas páginas de bibliotecas cuentan con un contacto general, y, en los casos en que sí hay acceso a redes sociales, los usuarios pueden usar estas páginas de manera activa a modo de referencia, aunque no esté establecida como tal por parte de la institución. Por lo tanto, no se observa una referencia estructurada con las herramientas específicas para ello. Los accesos al contacto bibliotecario digital son dispares, debido a la divergencia de desarrollos y diseños entre los sitios web, que a su vez se torna en un indicativo para considerar una homologación de presencia web de las bibliotecas en términos de estructura y navegación, para así contemplar el establecimiento de un sólo tipo de servicio de referencia digital.

A continuación, se describen las tres modalidades de comunicación encontradas en este subsistema de la unAm, detallando sus respectivas ventajas y desventajas.

\section{Estructura del servicio de referencia vía correo electrónico}

De las bibliotecas analizadas, se observó que en 11 casos se da el acceso a un contacto mediante correo electrónico, con una disposición textual de la dirección de correo. En otros tres casos, dicha cuenta de correo se encuentra vinculada con el sistema de correo automático del usuario. En todos los casos se encuentra este método de contacto como un apoyo general, no especializado.

Los contactos electrónicos aquí señalados podrían potencialmente cubrir (aunque sea de forma parcial) dichas necesidades. Y es que un aspecto indispensable de este método radica en su localización dentro de la pantalla, aunque en varias ocasiones se identificó que el contacto por correo no es localizable de manera efectiva.

Por otro lado, la única ventaja posible de una disposición textual del correo de contacto es que el usuario copie dicha dirección y la utilice en cualquier sistema de correo que desee; sin embargo, este es un método muy pobre y limitado. Su característica obvia es la asincronización, que otorga, tanto al usuario como al bibliotecario, el tiempo suficiente para elaborar y aterrizar sus respectivas preguntas y respuestas. Si se considera cualquier otro aspecto, este esquema no ofrece otras ventajas discernibles.

Las desventajas como referencia de esta modalidad de contacto se hacen patentes a primera vista: las posibilidades de un contacto directo o en tiempo real son nulas. No existe una flexibilidad razonable en la comunicación que permita un asesoramiento elaborado para implementar estrategias de búsqueda de información. En caso de requerir una atención especializada, ésta se tendría que llevar a cabo mediante una abundante serie de correos para tal efecto. 


\section{Estructura del servicio de referencia vía referencia social}

Se encontró que la disposición de contacto mediante red social se da como parte de la navegación global o dentro de un menú contextual. Regularmente, el acceso se da mediante botones iconográficos separados de otros elementos. El arreglo y estructura de estos botones son de gran importancia, porque si dicha configuración forma parte de una navegación global significa que estará presente durante todas las secciones del sitio, al darle una posición notable y el rol de verdadero servicio.

En cambio, cuando la configuración se encuentra dentro de una estructura de navegación contextual, el contacto mediante red social pasa a localizarse sólo en sectores selectos del sitio, como elemento relativo a dicha sección. Este es el caso de la mayoría de los sitios que cuentan con redes sociales. La razón principal de ello es que la red está vinculada como una cuenta social general de la institución y no dedicada a una consulta especializada a los usuarios de biblioteca.

\section{Estructura del servicio de referencia vía chat}

Como se mencionó en el tercer apartado, Referencia digital en la unAm, el sitio web de la Biblioteca Digital, se considera como un caso especial porque no pertenece a una biblioteca en particular, pero se encuentra vinculada desde varias bibliotecas y ofrece sus servicios a todo el Sistema Bibliotecario de la unAm. En su página web tiene incorporado un sistema de chat que funge de forma especializada como servicio de referencia digital. En la imagen 3 se muestra la ubicación de dicho sistema de chat dentro de la interfaz, así como un acercamiento al despliegue de la ventana.

Imagen 3. Ubicación del sistema de chat para consultas en BiDi-unam. Fuente: sitio web BiDi-unam, 2019.

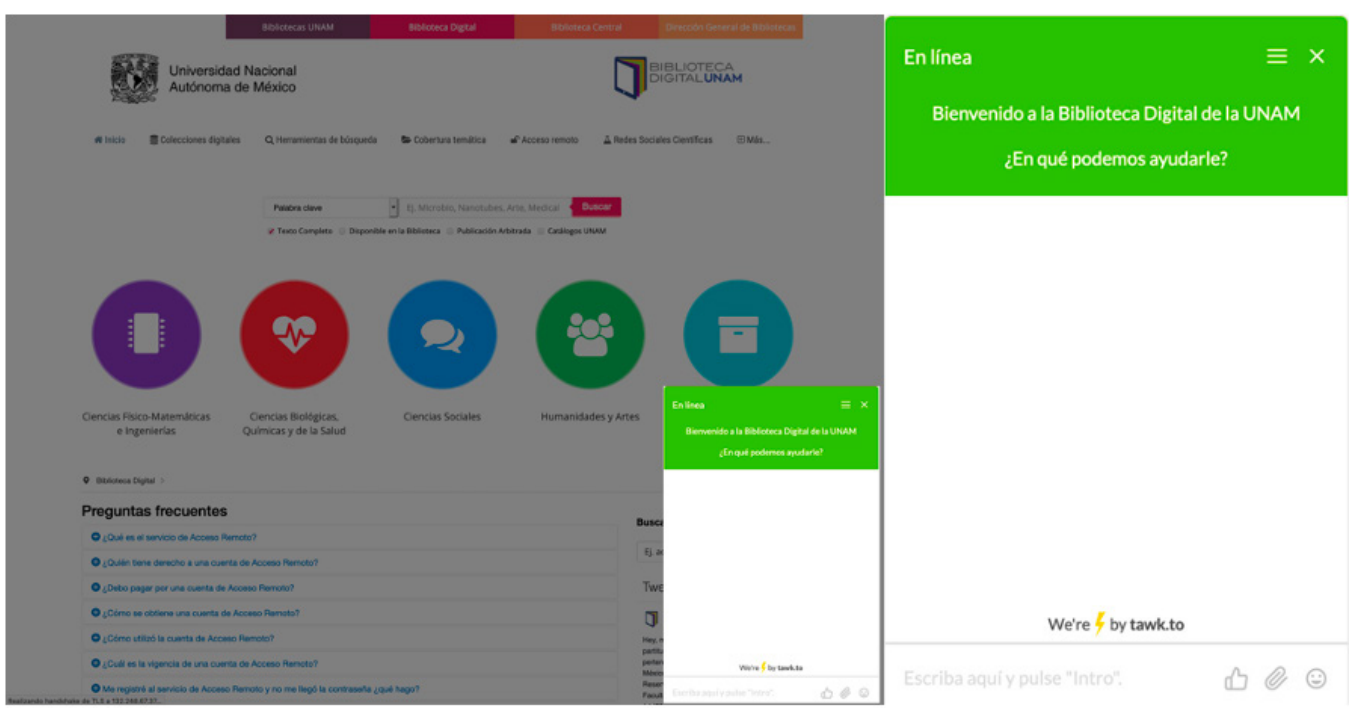

Este servicio de chat tiene un horario de atención de 8:00 a 21:00 horas, y cuando no se encuentra activo incorpora en su lugar un breve formulario de contacto para envío de preguntas y consultas. De esta manera, ofrece con ello atención 
Imagen 4. Formulario de contacto fuera de horario de servicio en BiDi-unam. Fuente: sitio web BiDi-unam, 2019.

Imagen 5. Herramientas contextuales del chat. Fuente: sitio web BiDi-unam, 2019.

asincrónica para no descuidar a los usuarios. En la imagen 4, se observan a detalle los campos que se tienen disponibles.

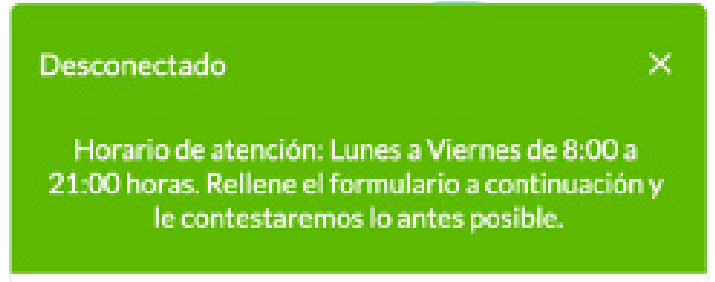

Este sistema se encuentra ubicado en la parte inferior derecha de la pantalla. Al abrir el sitio web se muestra como una pestaña, discreta pero visible, y al dar clic en ella se activa la ventana. Esta disposición es altamente efectiva debido a que se muestra al frente de la página de forma privilegiada, pero sin opacar o invadir el resto del sitio web para añadirse como un servicio independiente del sistema de navegación y presente en todas las secciones internas. Esto permite continuar la consulta de referencia sin importar la ubicación del usuario dentro de la página.

Al contemplar la estructura interna

Enviar

Weire 4 by tawk.to

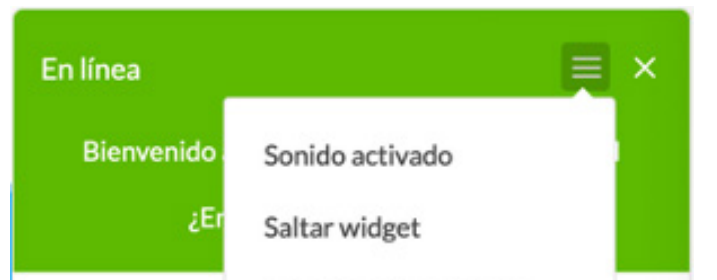

Final de esta sesión de chat

We're 4 by tawk.to

Escriba aquily pulse "Intro". del chat, como se muestra en la imagen 5, se observa que al abrirlo presenta una barra de encabezado que tiene una triple función. Por una parte, muestra mediante características textuales el estado activo o inactivo del servicio y un mensaje de bienvenida. Su siguiente función consiste en un menú contextual de tres opciones dedicadas a la administración de la sesión interna de la herramienta; una de ellas es de gran relevancia y está titulada "Saltar Widget", la cual permitir "sacar" el chat de la ventana principal del explorador de internet. Con esta función se logra una independencia del servicio sin importar la ubicación o estado del sitio de la biblioteca digital, y se hace posible cerrar dicha ventana principal y mantener abierta la sesión de referencia.

En lo que concierne al manejo de botones, se llevan al mínimo y ofrecen por ello una experiencia limpia. Dentro de las herramientas de escritura, hay tres íconos claros con las opciones de insertar los emoticones de Me gusta/No me gusta, además de la capacidad de adjuntar archivos. 
Una vez iniciada la consulta se da una sesión constante durante toda la navegación, con facilidad de uso, y atención sincrónica en tiempo real durante los horarios de servicio (ver imagen 6). Incluso, no se descuida al usuario fuera de horario de servicio al ofrecer una herramienta asincrónica adicional. Por todo lo anterior, se puede concluir que esta página cuenta con una adecuada ubicación y una apropiada aplicación técnica del sistema.

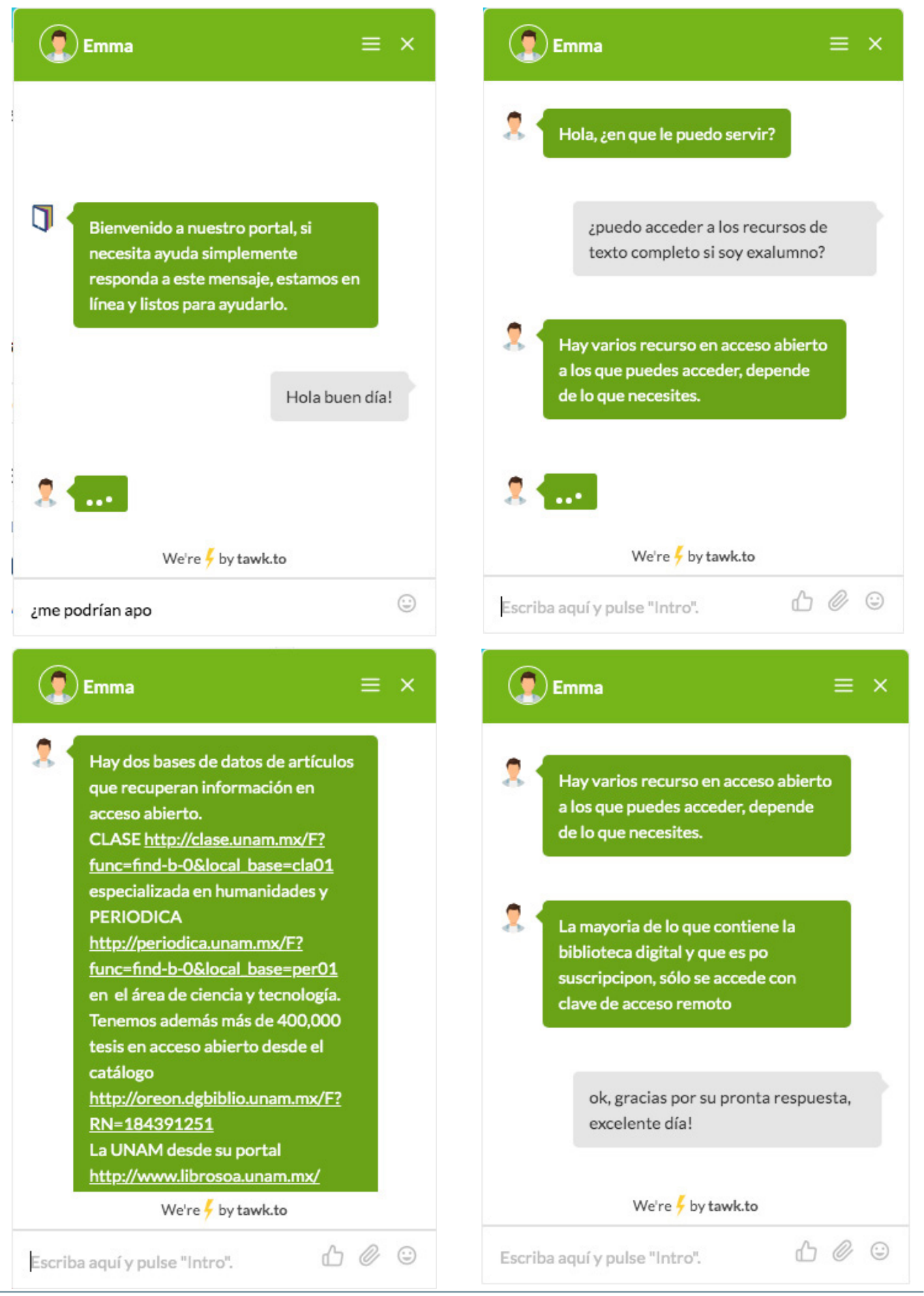


"Panorama del servicio de referencia digital en bibliotecas de la UNAM"

Bardo Javier García Martínez

Vol. 21, Núm. 6, noviembre-diciembre 2020

Revista Digital Universitaria

Esta modalidad de referencia no se implementa en las bibliotecas de manera individual; por lo tanto, no hay una consulta de referencia especializada para cada disciplina. En este caso, lo difícil es que el bibliotecario detrás de dicho servicio pueda solventar esa tipología de consultas. En estos escenarios lo más que se puede ofrecer son los datos de contacto correspondiente. Esto nos regresa a la problemática actual aquí planteada: el usuario queda a merced del tipo de contacto que se tenga al final de este proceso.

\section{Reflexiones finales}

Al presentar aquí el panorama y parámetros de uso que comprenden la configuración de los distintos accesos a una posible referencia digital, dentro del subsistema bibliotecario en cuestión, se evidencian las carencias y características que existen en este entorno. Todo ello aporta una serie de posibilidades que permitirán generar un diagnóstico formal y más detallado de la tecnología digital utilizada. Hay que aclarar que, si se desea ahondar más en dicho aspecto técnico, sería necesario conocer a profundidad las capacidades humanas y los alcances tecnológicos con los que cuenta cada biblioteca. En todo caso, se obtendría la materia prima para realizar otro tipo de análisis.

Respecto al presente artículo y considerando la experiencia desde la perspectiva como usuario, presente en este análisis, es posible sugerir algunas recomendaciones al respecto:

1. En los casos en que se recurre al uso de vínculos textuales hacia un servicio de atención al usuario, éstos deben mostrarse de manera intuitiva. Especialmente si el servicio se proporciona mediante una cuenta de redes sociales, pues en esta situación es muy importante cuidar que los botones sean claros al indicar, en efecto, que se está ofreciendo información o algún contacto para asuntos generales o, si en todo caso, se utiliza como un medio de servicio de referencia. La diferencia en este escenario es importante, ya que dicho arreglo separa las consultas básicas y generales de las especializadas.

2. Uno de los aspectos más importantes a cuidar es la urgencia de una homologación de la estructura web de las bibliotecas de este subsistema. De seguir el ejemplo de BiDi-unam, sería posible la integración de un chat como referencia digital. De hecho, en términos de estructura, este sitio resulta ejemplar, porque además cuenta con una estructura de navegación jerárquica, a dos y tres niveles de profundidad, que consta de tres menús claramente diferenciados.

3. Resulta interesante la manera en que un sitio con una distribución de contenidos tan abundantes pueda priorizar el servicio de referencia sin invadir de manera alguna la organización y navegación establecida. Se enfrenta, por un lado, al reto tecnológico de programación e integración y, por el otro, a la designación de personal adecuado para la atención especializada, así como la familiarización técnica con la 
herramienta. Todas estas competencias en conjunto requieren de una labor en equipo por sus características.

Existen una multitud de elementos para lograr una referencia adecuada dentro de las bibliotecas aquí exploradas. Los factores que probablemente evitan que un chat sea implementado de manera individual comienzan, por un lado, con la capacidad técnica reflejada en la disparidad de desarrollo que se da en cada sitio de biblioteca, y por el otro, con el aspecto humano que recae en la capacidad y disponibilidad de personal en cada sitio web. El presente documento es una visita al panorama general de la unAm y, en consecuencia, se espera que sirva de antecedente para acercar a uno de los servicios de información más importantes a los usuarios de la Universidad.

\section{Referencias}

- Bibliotecas unam. (2019). Licenciatura y Posgrado, Localiza la biblioteca de la Escuela o Facultad de tu interés. http://www.bibliotecas.unam.mx/index.php/licenciaturay-posgrado

* Biblioteca Digital unam. (2019). Dirección General de Bibliotecas de la Universidad Nacional Autónoma de México. https://bidi.unam.mx/

* González, N. (2012). Servicios de referencia en bibliotecas universitarias: Tendencias y plan de marketing. El profesional de la Información, 21(6), 567-576. Dol: http://dx.doi.org/10.3145/epi.2012.nov.03

- International Federation of Library Associations and Institutions. (2002). IFLA Digital Reference Guidelines. https://www.ifla.org/publications/ifla-digital-referenceguidelines?og=8708

* Katz, L. (2002). Digital Reference Services. The Haworth Press.

- Martínez del Prado, A. (2008) Criterios para desarrollar servicios de referencia virtual [tesis de maestría, Facultad de Filosofía y Letras, Universidad Nacional Autónoma de México]. http://oreon.dgbiblio.unam.mx/F?RN=933918803

- Reference and User Services Association. (2017). Professional Competencies for Reference and User Services Librarians. American Library Association. http://www. ala.org/rusa/resources/guidelines/professional

\section{Cómo CITAR ESTE ARTículo}

* García Martínez, Bardo Javier. (2020, noviembre-diciembre). Panorama del servicio de referencia digital en bibliotecas de la unAM. Revista Digital Universitaria (RDU), 21(6). Dol: http://doi.org/10.22201/cuaieed.16076079e.2020.21.6.12 


\section{Anexo}

\section{Panorama del servicio de referencia digital en bibliotecas de la UNAM}

\begin{tabular}{|c|c|}
\hline Entidad & Bibliotecas \\
\hline $\begin{array}{l}\text { Escuela Nacional de Artes } \\
\text { Cinematográficas }\end{array}$ & Biblioteca Escuela Nacional de Artes Cinematográficas \\
\hline $\begin{array}{l}\text { Escuela Nacional de Enfermería } \\
\text { y Obstetricia }\end{array}$ & Biblioteca "Graciela Arroyo de Cordero" \\
\hline $\begin{array}{l}\text { Escuela Nacional de Estudios } \\
\text { Superiores. Unidad León, Gto. }\end{array}$ & $\begin{array}{l}\text { Biblioteca Escuela Nacional de Estudios Superiores. Unidad } \\
\text { León, Gto. }\end{array}$ \\
\hline $\begin{array}{l}\text { Escuela Nacional de Estudios } \\
\text { Superiores. Unidad Morelia, } \\
\text { Michoacán }\end{array}$ & $\begin{array}{l}\text { Biblioteca Escuela Nacional de Estudios Superiores. Unidad } \\
\text { Morelia, Michoacán }\end{array}$ \\
\hline $\begin{array}{l}\text { Escuela Nacional de Lenguas, } \\
\text { Lingüística y Traducción }\end{array}$ & Biblioteca "Stephen A. Bastien" \\
\hline $\begin{array}{l}\text { Escuela Nacional de Trabajo } \\
\text { Social }\end{array}$ & Centro de Información y Servicios Bibliotecarios \\
\hline Facultad de Arquitectura & Biblioteca "Lino Picaseño" \\
\hline Facultad de Artes y Diseño & $\begin{array}{l}\text { Centro de Documentación "Prof. José Ma. Natividad Correa } \\
\text { Toca" }\end{array}$ \\
\hline Facultad de Ciencias & Biblioteca "Ricardo Monges López" \\
\hline $\begin{array}{l}\text { Facultad de Ciencias Políticas y } \\
\text { Sociales }\end{array}$ & Biblioteca "Isidro Fabela" \\
\hline $\begin{array}{l}\text { Facultad de Contaduría y } \\
\text { Administración }\end{array}$ & Biblioteca "C. P. Alfredo Adam Adam" \\
\hline Facultad de Derecho & Biblioteca "Antonio Caso" \\
\hline Facultad de Economía & Biblioteca "Ramón Ramírez Gómez" \\
\hline $\begin{array}{l}\text { Facultad de Estudios } \\
\text { Superiores Acatlán }\end{array}$ & Centro de Información y Documentación \\
\hline $\begin{array}{l}\text { Facultad de Estudios } \\
\text { Superiores Aragón }\end{array}$ & Biblioteca "Jesús Reyes Heroles" \\
\hline $\begin{array}{l}\text { Facultad de Estudios } \\
\text { Superiores Cuautitlán }\end{array}$ & $\begin{array}{l}\text { - Biblioteca Facultad de Estudios Superiores } \\
\text { Cuautitlán Campo } 1 \\
\text { - Biblioteca Facultad de Estudios Superiores } \\
\text { Cuautitlán Campo } 4\end{array}$ \\
\hline $\begin{array}{l}\text { Facultad de Estudios } \\
\text { Superiores Iztacala }\end{array}$ & Unidad de Documentación Científica \\
\hline $\begin{array}{l}\text { Facultad de Estudios } \\
\text { Superiores Zaragoza }\end{array}$ & $\begin{array}{l}\text { - Biblioteca Facultad de Estudios Superiores } \\
\text { Zaragoza Campo } 1 \\
\text { - Biblioteca Facultad de Estudios Superiores } \\
\text { Zaragoza Campo } 2\end{array}$ \\
\hline Facultad de Filosofía y Letras & Biblioteca "Samuel Ramos" \\
\hline Facultad de Ingeniería & Biblioteca "Ing. Antonio Dovalí Jaime" \\
\hline Facultad de Medicina & Biblioteca. Facultad de Medicina \\
\hline
\end{tabular}

Tabla 1. Bibliotecas por entidad en el subsistema de Licenciatura y Posgrado de la Universidad Nacional Autónoma de México.

Fuente: sitio web BiDi-unam, 2020. Fuente: sitio web del Sistema Bibliotecario de la UnAM (Bibliotecas unam, 2019). 


\begin{tabular}{|l|l|}
\hline \multicolumn{2}{|l|}{ Facultad de Medicina } \\
Veterinaria y Zootecnia & - Biblioteca Centro de Enseñanza, Inv. y Ext. en \\
& Ganadería Tropical \\
& - Biblioteca Centro de Enseñanza, Inv. y Ext. en \\
& Producción Ovina \\
& - Biblioteca Centro de Enseñanza, Inv. y Ext. en \\
& Producción Avícola \\
& - Biblioteca Centro de Enseñanza, Inv. y Ext. en \\
& Producción Porcina \\
& - Biblioteca "M.V. José de la Luz Gómez" \\
& - Biblioteca Centro de Enseñanza, Inv. y Ext. en \\
& Producción Animal en Altiplano \\
\hline Facultad de Música & Biblioteca "Cuicamatini" \\
\hline Facultad de Odontología & - Biblioteca "Dr. Ignacio Aguilar Álvarez" \\
& - Biblioteca "Dr. Barnet M. Levy" \\
\hline Facultad de Psicología & - Biblioteca "Dra. Graciela Rodríguez Ortega" \\
& - Centro de Documentación "Dr. Rogelio Díaz \\
& Guerrero" \\
\hline Facultad de Química & Biblioteca de Estudios Profesionales de Facultad de Química \\
\hline
\end{tabular}

Tabla 2. Características de las tipologías de contacto encontradas. Fuente: elaboración propia, 2019.

1 Esta herramienta se considera como un caso muy especial, ya que no se encuentra en ninguno de los 35 sitios web de bibliotecas; sin embargo, se plantea aquí debido a que en diversos sitios se ofrecen vínculos dirigidos al sitio web de la Biblioteca Digital de la UnAm (BiDi- unam), y es ahí donde se proporciona la herramienta de chat, y el objetivo de dicho sitio se encuentra dirigido a toda la comunidad UNAM.

Tabla 3. Tipos de acceso mediante correo electrónico. Fuente: elaboración propia, 2019.

Tabla 4. Tipos de acceso mediante redes sociales. Fuente: elaboración propia, 2019.

\begin{tabular}{|c|c|c|}
\hline $\begin{array}{c}\text { Sitios } \\
\text { web de } \\
\text { bibliotecas }\end{array}$ & Modalidad de contacto & Características \\
\hline 14 & Vínculo a correo electrónico & $\begin{array}{l}\text { Dirección de correo electrónico vinculado a la } \\
\text { apertura predefinida del cliente de correo en } \\
\text { el sistema operativo del usuario y dirección } \\
\text { de correo electrónico de manera textual (para } \\
\text { copiar y pegar) }\end{array}$ \\
\hline 8 & Miniformulario de contacto & $\begin{array}{l}\text { Contacto asincrónico, mensaje con los campos } \\
\text { requeridos de: nombre, correo, mensaje, } \\
\text { seguridad mediante Captcha }\end{array}$ \\
\hline 13 & Redes sociales & $\begin{array}{l}\text { Contacto con mensaje directo en chat social, } \\
\text { comunicación mediante posts generales. }\end{array}$ \\
\hline 1 & Chat $^{1}$ & $\begin{array}{l}\text { Horario de atención sincrónica de lunes } \\
\text { a viernes de 8:00 a 21:00 horas. Atención } \\
\text { asincrónica fuera de horario mediante } \\
\text { formulario de contacto con los campos de: } \\
\text { nombre, correo, mensaje, sistema } \\
\text { desarrollado mediante twak.to }\end{array}$ \\
\hline 11 & $\begin{array}{l}\text { No ofrecen método de } \\
\text { contacto digital }\end{array}$ & \\
\hline
\end{tabular}

\section{Sitios web de Modalidad de contacto: correo electrónico} bibliotecas textual

\begin{tabular}{|c|l|}
\hline 11 & Sólo dirección de correo textual \\
\hline 3 & Vínculo a sistema de correo predefinido \\
\hline
\end{tabular}

Bibliotecas Modalidad: redes sociales

\begin{tabular}{|l|l|}
\hline 9 & Sólo vinculación a la cuenta \\
\hline 4 & Chat preparado con preguntas de bienvenida genéricas \\
\hline 0 & Chat preparado con preguntas de referencia especializada \\
\hline
\end{tabular}

